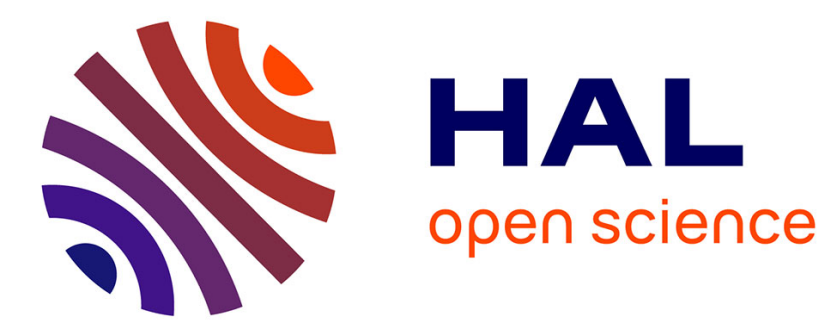

\title{
Experimental study of electrical FitzHugh-Nagumo neurons with modified excitability
}

Stéphane Binczak, Sabir Jacquir, Jean-Marie Bilbault, Victor B. Kasantsev, Vladimir I. Nekorkin

\section{- To cite this version:}

Stéphane Binczak, Sabir Jacquir, Jean-Marie Bilbault, Victor B. Kasantsev, Vladimir I. Nekorkin. Experimental study of electrical FitzHugh-Nagumo neurons with modified excitability. Neural Networks, 2006, 19 (5), pp.684-693. 10.1016/j.neunet.2005.07.011 . hal-00584220

HAL Id: hal-00584220

https://u-bourgogne.hal.science/hal-00584220

Submitted on 7 Apr 2011

HAL is a multi-disciplinary open access archive for the deposit and dissemination of scientific research documents, whether they are published or not. The documents may come from teaching and research institutions in France or abroad, or from public or private research centers.
L'archive ouverte pluridisciplinaire HAL, est destinée au dépôt et à la diffusion de documents scientifiques de niveau recherche, publiés ou non, émanant des établissements d'enseignement et de recherche français ou étrangers, des laboratoires publics ou privés. 


\title{
Experimental study of electrical MFHN neurons
}

\author{
S. Binczak ${ }^{\mathrm{a}}$, S. Jacquir ${ }^{\mathrm{a}}$, J.M. Bilbault ${ }^{\mathrm{a}}$, V.B. Kazantsev ${ }^{\mathrm{b}}$ and V.I. Nekorkin ${ }^{\mathrm{b}}$ \\ a) Laboratoire LE2I, CNRS UMR 5158, \\ Aile des Sciences de l'Ingénieur, \\ Université de Bourgogne, \\ BP 47870, Dijon Cedex, France \\ b) Institute of Applied Physics of RAS, \\ 46 Uljanov str., \\ 603950 Nizhny Novgorod, Russia
}

June 23, 2004

\begin{abstract}
We present an electronical analogue circuit modelling a FitzHugh-Nagumo neuron with a modified excitability. To characterize this basic cell, the bifurcation curves between stability with excitation threshold, bistability and oscillations are investigated. An electrical circuit is then proposed to realize an unidirectional coupling between two cells, mimicking an inter-neuron synpatic coupling. In such a master-slave configuration, we show experimentally how the coupling strength control the dynamic of the slave neuron, leading to frequency locking, chaotic behavior and synchronization. These phenomena are then studied by phase map analysis. The architecture of a possible neural network is then described introducing different kind of coupling between neurons.
\end{abstract}

\section{Introduction}

As biological neurons have been well studied over the previous century, there is nowadays a strong interest in realizing electrical networks to reproduce the activity of biological neurons. Accurate models are usually used to describe biological neural network, such as Hodgkin and Huxley (HH) [1], and are subject to intensive studies $[2,3]$ in engineering science. Never- theless, mathematicians often study the 2-component FitzHugh-Nagumo (FHN) model [4, 5], as a simplified version of the $\mathrm{HH}$ one. This set of equations leads indeed to analytical studies, enlightening the nonlinear dynamical mechanisms generating complex patterns in the neural information process [6].

Although these differential equations are used to model the nerve membrane $[6,7]$, we suggest to describe overall activity of the neuron by them. In this reductive approach, we propose an experimental electronic implementation of a neuron based on the FitzHugh-Nagumo (FHN) equation with modified excitability to conceive the electronic MFHN neuron [8].

In the first part, the electronic neuron is presented and characterized by its experimental bifurcation curves, showing that this MFHN circuit leads to complex dynamics of travelling waves $[9,10]$ emerging from saddle homoclinic loop bifurcations.

In the second part, we use the MFHN circuit as a basic cell to realize a master-slave configuration. Two cells are coupled in an unidirectional manner, which would correspond to two neurons coupled synaptically. After the presentation of the electronic circuit giving this coupling, we discuss the experimental conditions for which the master dynamics controls the 
excitability of the slave neuron leading to a shift of bifurcation curves, a variation of interspike frequencies or a phenomenon of intermittency route to chaos. Finally, we present the architecture of a possible experimental network including different electrical realizations of neuronal coupling.

\section{Experimental description of one cell}

\subsection{Electrical circuit}

The nonlinear circuit, as sketched in Fig. 1, can be described as follows: Part (A) is a parallel association of three different branches, two of them being resistive and commuted by silicium diodes $(V d=0.6 \mathrm{~V})$ while the third one is a negative resistor obtained with an operational amplifier. Due to diodes' com-

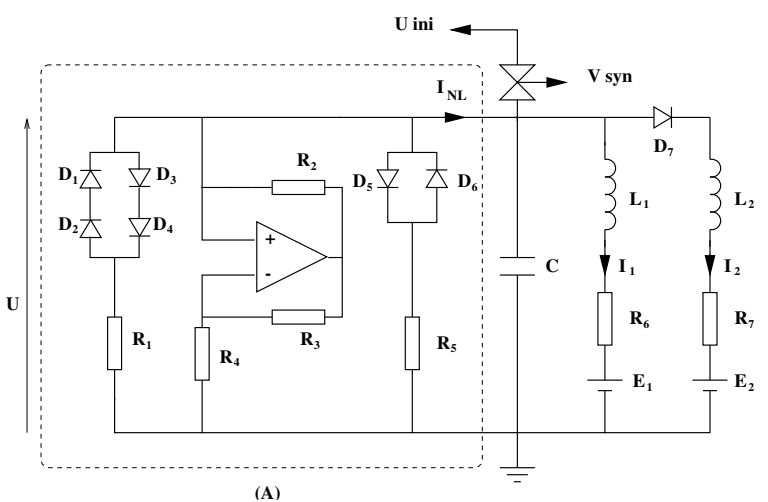

Figure 1: Diagram of the nonlinear circuit

muting behaviour, the resulting $\mathrm{I}-\mathrm{V}$ characteristic is nonlinear and can be modelled by a cubic polynomial function for an appropriate set of parameters so that

$$
I_{N L}=f(U)=\frac{1}{R_{0}}\left[U-\frac{\gamma^{2} U^{3}}{3}\right],
$$

where $U$ and $I_{N L}$ are respectively the voltage and the corresponding current. The parameters $R_{0}$ and $\gamma$ are obtained by a fitting approximation, e.g. by least mean square's method. As illustrated in Fig. 2 , we obtain a good match between experimental results and equation (1) by setting $R_{0}=1010 \Omega$ and $\gamma=1.138 V^{-1}$. This nonlinear resistor is in parallel

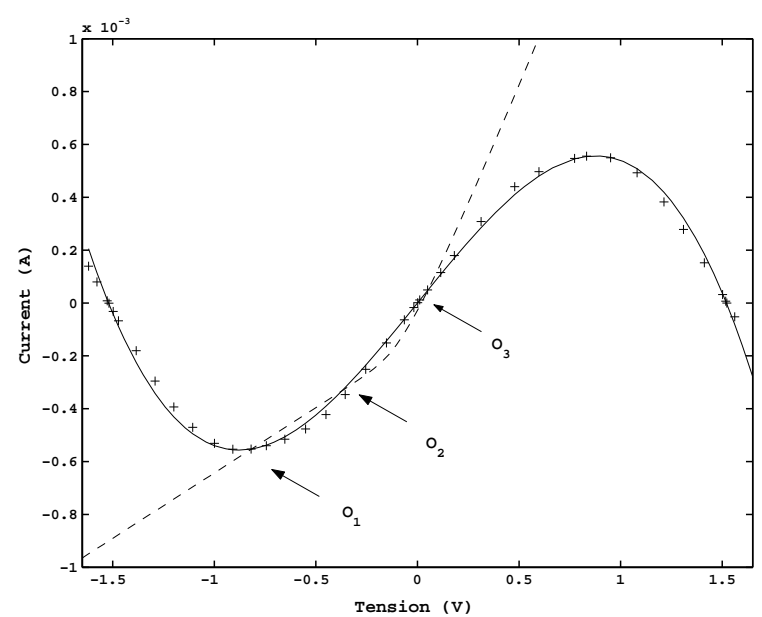

Figure 2: Experimental I-V characteristic (+ symbols) of part (A). Continuous line: $I_{N L}=f(U)$ from eq. (1) with $R_{0}=1010 \Omega$ and $\gamma=1.138 V^{-1}$. Dashed line: Experimental I-V characteristic $I_{1}+$ $I_{2}=h(U)$ with $R_{6}=2021 \Omega, R_{7}=690 \Omega, L_{1}=$ $10.2 \mathrm{mH}, L_{2}=3.5 \mathrm{mH}$ and $E_{1}=0.4 \mathrm{~V}$

with a capacitance and two branches in parallel including inductances, resistances and voltage sources, one of them being commuted by a silicium diode so that setting the conditions $\frac{R_{6}}{L_{1}}=\frac{R_{7}}{L_{2}}, E_{2}=-V d$, and using a piecewise linear I-V description for diode $D_{7}, I_{2}=0$ if $U<0$. Therefore, using Kirchhoff's laws, the system of equations can be expressed in a normalized way by :

$$
\left\{\begin{array}{l}
\frac{d V}{d \tau}=\left[V-\frac{V^{3}}{3}\right]-W \\
\frac{d W}{d \tau}=\varepsilon[g(V)-W-\eta]
\end{array}\right.
$$

Where $V=\gamma U$ and $W=\gamma R_{0}\left(I_{1}+I_{2}\right)$ correspond, in biological terms, to the membrane voltage and the recovery variable; $\tau=\frac{t}{R_{0} C}$ is a rescaled time, $\varepsilon=\frac{R_{0} R_{6} C}{L_{1}}$ the recovery parameter and $\eta=\gamma \frac{R_{0}}{R_{6}} E_{1}$ a bifurcation parameter. $g(V)$ is a piecewise linear 
function, $g(V)=\alpha V$ if $V \leq 0$ and $g(V)=\beta V$ if $V>0$ where $\alpha=\frac{R_{0}}{R_{6}}$ and $\beta=\frac{L_{1}+L_{2}}{L_{2}} \frac{R_{0}}{R_{6}}$ control the shape and location of the $\mathrm{W}$-nullcline [8].

Note also that the initial condition $U_{i n i}$ can be loaded in the neuron via an analogue commutator controlled by a periodic signal $V_{s y n}$.

\subsection{Experimental bifurcation curves of MFHN circuit}

This section presents different dynamics of the MFHN neuron [8]. In the case, $\alpha=\beta$, the system corresponds to the standard FitzHugh-Nagumo (FHN) equation where the recovery variable is driven by a linear function of the "membrane voltage". In the general case $\alpha \neq \beta$, equation (2) exhibits much more complex dynamics. The phase portrait is very similar to the one occurring from the modified Morris-Lecar (ML) equations $[11,7]$ proposed to model barnacle muscle fibres and pyramidal cells. Considering the case $\alpha=0.5$ and $\beta=1.96$, experimental bifurcation curves in the parameter plane $(\eta, \varepsilon)$ is presented in Fig. 3. Several results are to be distinguished de-

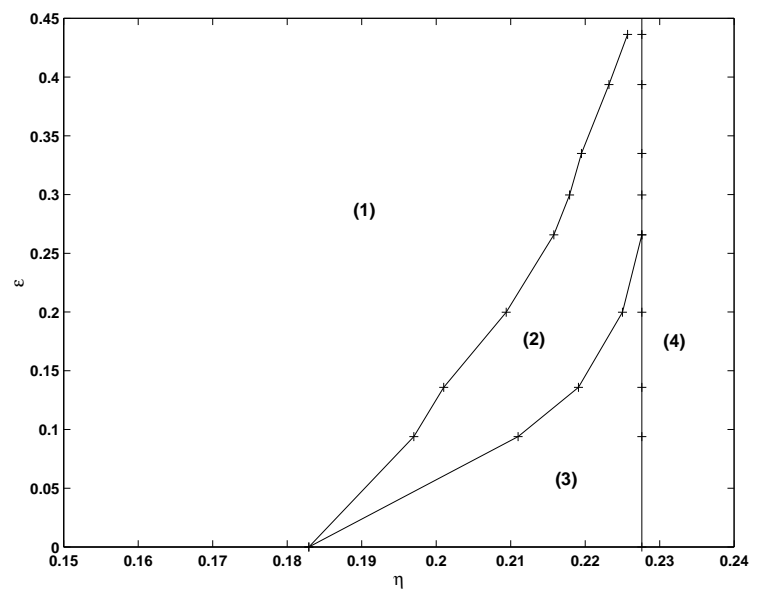

Figure 3: Experimental bifurcation curves in the dimensionless plane $(\eta, \varepsilon)$.

pending on the location of the nullcline points and on initial conditions. In domain (1), the systeme has one stable and unstable fixed point, while one point is a saddle. Then, if a perturbation of the rest state, $O_{1}$, is large enough so that it lies between the points $\mathrm{O}_{2}$ and $\mathrm{O}_{3}$, the system responds with an excitation pulse, as illustrated in Fig. 4. The upper inset shows

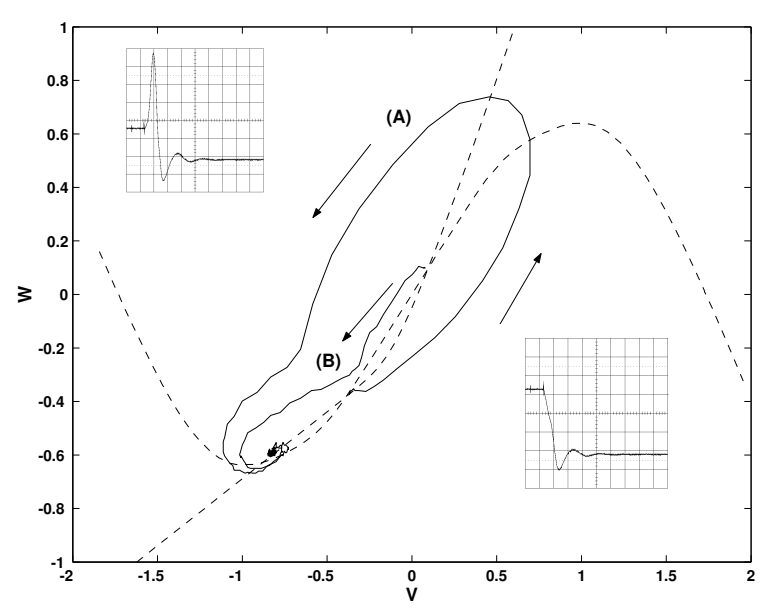

Figure 4: Dimensionless phase plane (V,W) showing stability with excitation threshold corresponding to domain (1) of Fig.3 with $\eta=0.19$ and $\varepsilon=0.2$ $\left(E_{1}=0.332 V\right.$ and $\left.C=1 n F\right)$. Insets: Excitation pulse (upper inset); perturbation reaching resting state (lower inset). Parameters of insets: abscissa $20 \mu \mathrm{s} /$ div ; ordinate $225 \mathrm{mV} /$ div .

an excitation pulse while the bottom one shows a perturbation reaching the resting state; the main figure gives the respective experimental phase portrait for the both cases. Domain (2) corresponds to the bistability case characterized by the existence of a stable fixed point and a stable limit cycle that has appeared from a big homoclinic loop bifurcation. Then, the model exhibits oscillations if the perturbation is large enough, as illustrated in Fig. 5. The inset shows a spiking train of pulses corresponding to the limit cycle in the experimental phase portrait. Otherwise, it decays to the rest state. In domain (3), the fixed point looses stability via a subcritical Andronov-Hopf bifurcation and only oscillations occur in the model, which are similar to the spiking train of pulses. Note that in the region (2), another saddle homoclinic loop bifurcation has taken place leading to a small unsta- 


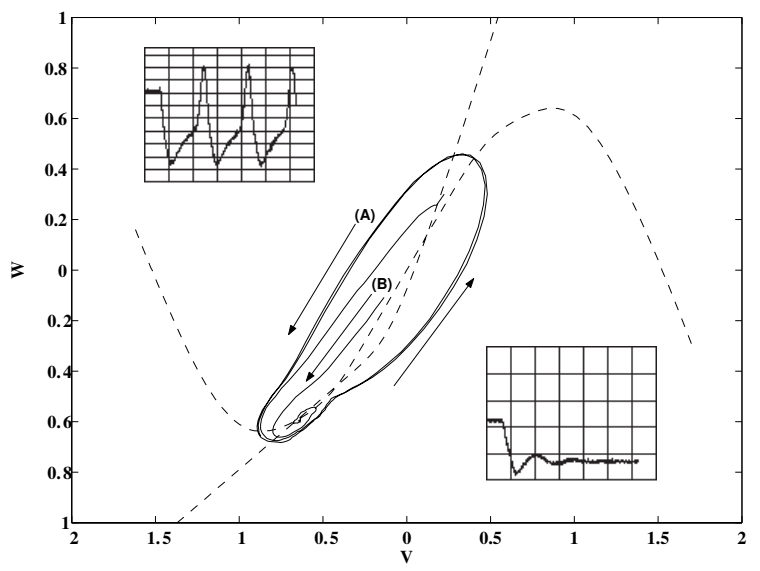

Figure 5: Dimensionless phase plane (V,W) showing bistability corresponding to domain (2) of Fig. 3 with $\eta=0.226$ and $\varepsilon=0.2\left(E_{1}=0.3981 V\right.$ and $C=1 n F)$. Inset: Spiking train of pulses corresponding to limit cycle in experimental phase portrait. Parameters of inset: abscissa $20 \mu \mathrm{s} / \mathrm{div}$; ordinate $195 \mathrm{mV} /$ div.

ble limit cycle near the fixed point. Note also that, contrary to standard FHN, arbitrarily long interspike intervals can be found theoretically, as the two lower equilibrium points are merging. Finally, in domain (4), a single unstable fixed point exists leading to oscillations. In domains (2) and (3), the frequency of the oscillations is controlled by the parameters $\eta$ and $\varepsilon$, leading to a wide range of values, as illustrated in Fig. 6.

In the following section, we study the dynamical behaviour of a network composed of two neurons connected unilaterally.

\section{Unidirectional coupling of two cells}

The neurons communicate mainly between them through specialized devices called synapses via chemical messages. The chemical synapse transmits the impulse unidirectionally. Therefore, it is interesting to conceive and to realize an electrical circuit in-

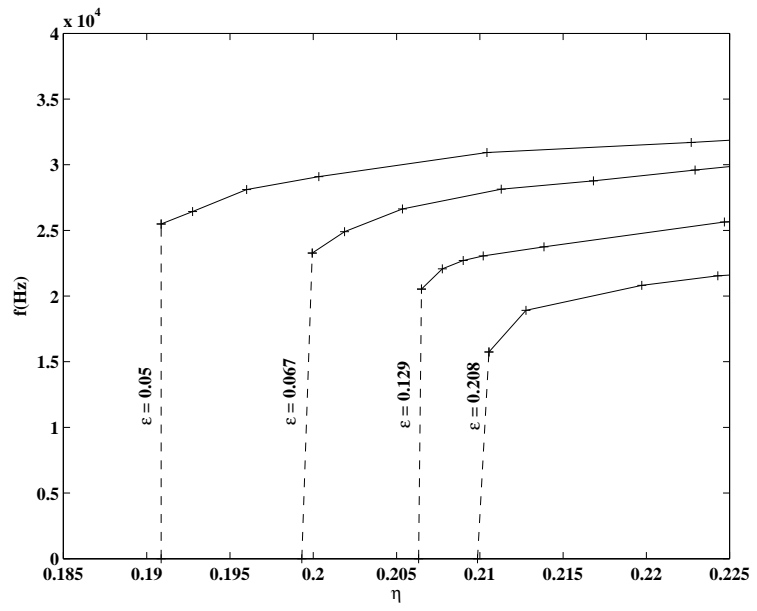

Figure 6: Frequency of an oscillating neuron in function of the parameters $\eta$ and $\varepsilon$. The dot lines correspond to a jump from the resting state to the oscillating one.

cluding the same features as the synaptical coupling. We present in Fig. 7 the unidirectional coupling between two cells leading to a master-slave configuration where $N_{i}(i=1,2)$ are described by the circuit of Fig. 1. Let us introduce D the coupling parame-

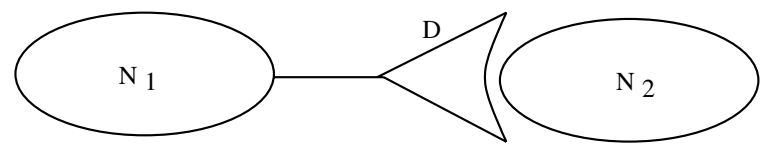

Figure 7: Coupling between two cells $N_{1}$ and $N_{2}$.

ter (synaptic strength). Its circuit, as illustrated in the Fig. 8, includes an adder-inverter, an inverter and then a follower. $U 1$ (resp. U2) is the voltage capacitor of the cell $N 1$ (resp. N2). The value of the resistor $\mathrm{R}$ is fixed to $100 \mathrm{k} \Omega$, which is large compared to the other components so that the current going though $2 R$ is negligible, $R a=10 \mathrm{k} \Omega$, while $R c$ is a detuning parameter which allows to control the coupling parameter value. Note also that the initial condition can be loaded in the neuron via an analogue commutator controlled by $V_{\text {syn }}$. Although it is usual to study a system with normalized variables, it 


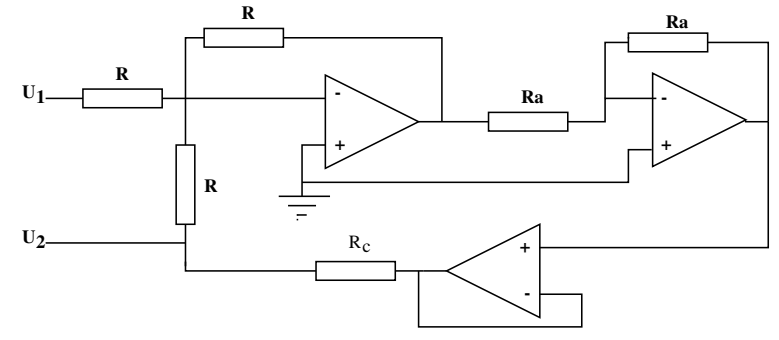

Figure 8: Unidirectional coupling circuit.

is more convenient to describe electrical circuits with experimental variables. Therefore, we will keep both variables (as normalized $V_{1}$ and experimental $U_{1}$ ) in the following of this paper. Using Kirchoff's laws, the normalized equations corresponding to the coupling between two MFHN neurons in a master-slave configuration can be expressed by:

$$
\left\{\begin{array}{l}
\frac{d V_{i}}{d \tau}=\left[V_{i}-\frac{V_{i}^{3}}{3}\right]-W_{i}+D V_{1} \delta_{2, i} \\
\frac{d W_{i}}{d \tau}=\varepsilon_{i}\left[g\left(V_{i}\right)-W_{i}-\eta_{i}\right]
\end{array}\right.
$$

with $i=\{1,2\}, D=\frac{R_{0}}{R_{c}}$, and where $\delta_{2, i}$ is a Kronecker symbol, so that $\delta_{2,1}=0$ and $\delta_{2,2}=1$.

Therefore, the two neurons are coupled so that a part of current weighted by $D$ via $R$, and generated by $N_{1}$ is included in $N_{2}$. The two neurons are initially set to voltage $U_{1} i n i$ and $U_{2} i n i$, due to the analogue commutators controlled by voltage $V_{\text {syn }}$. When the initial conditions are loaded, these commutators are switched off while the two neurons are connected via a third commutator controlled by $\overline{V_{s y n}}$. Note that the time delay between the two neurons has not been taken into account in this circuit, a master-slave configuration rendering it unnecessary.

\subsection{The master in a resting state}

When the voltage is so that $V_{1}$ is constant (the cell $N_{1}$ is in a resting state), the dynamics of the slave cell is given by eqs. (2) with effective bifurcation parameter

$$
\overline{\eta_{2}}=\eta_{2}-D V_{1}^{*}
$$

where $V_{1}^{*}<0$ is the voltage at stable resting state of the master. Therefore, it implies a modification of the excitability of the cell $N_{2}$ corresponding to a shift in the $(\eta, \varepsilon)$ plane illustrated on Fig. 9. The parameters are taken so that, when $D=0$, the master neuron $N_{1}$ lies in domain (1), while the slave neuron $N_{2}$ is in domain (3) and generates a spiking train of pulses. When the unilateral coupling is increased and reaches a critical value, the neuron $N_{2}$ ceases to oscillate and stays in the resting state, meaning that the slave neuron has been moved from domain (3) to domain (1) of Fig. 3. This ability of neuron $N_{1}$ to inhibit neuron $N_{2}$ corresponds to the shift predicted by equation (4): As $V_{1}^{*}<0$, increasing $D$ implies to increase $\overline{\eta_{2}}$ and therefore the bifurcation curves of Fig. 3 are translated along abscissa, while the value of $\eta_{2}$ defined by the electrical parameters of neuron $N_{2}$ has not been changed. This result suggests that, for a defined activity of a slave neuron, the strength of this unilateral coupling should be above a critical value to give to the master neuron the control on the slave neuron. In Fig. 9, experimental values $\left(D, \overline{\eta_{2}}\right)$

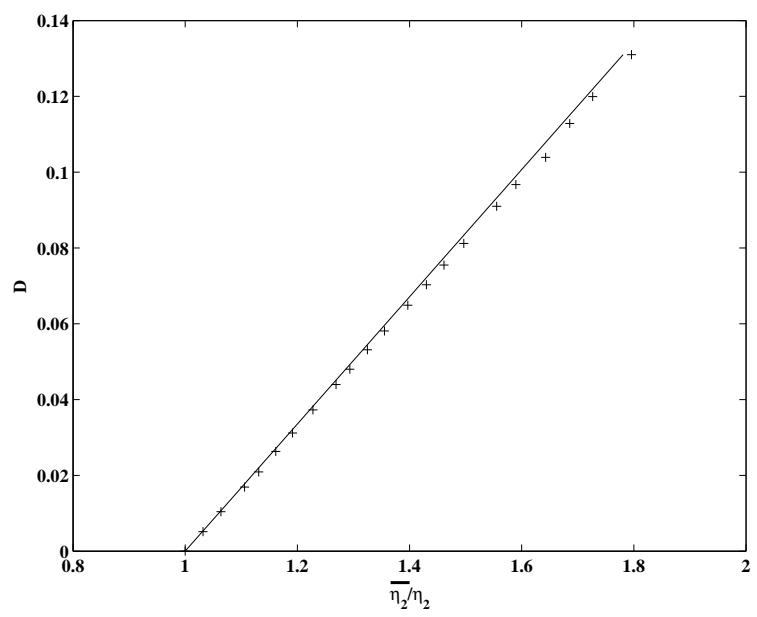

Figure 9: Shifted bifurcation curve of the slave neuron $N_{2}$ between domains (1) and (3). Parameters: Master neuron $N_{1}: \alpha_{1}=0.5, \beta_{1}=1.96, \epsilon_{1}=0.01$, $\eta_{1}=0.108$ leading to $V_{1}=-1.05$ (i.e. $U_{1}=$ $-921 \mathrm{mV})$. Slave neuron $N_{2}: \alpha_{2}=0.5, \beta_{2}=1.96$, $\epsilon_{2}=0.01$ 
correspond to the shifted bifurcation curve between domains (1) and (3) of the neuron $N_{2}$ with $\varepsilon_{2}=0.01$ and when the master neuron $N_{1}$ lies in domain (1) in a resting state so that $V_{1}^{*}=-1.05$. Comparison shows a good match between experimental results $(+)$ and equation (4) (continuous line), validating the unilateral coupling circuit.

\subsection{The master in a spiking regime}

In this section, we present some results when the master is in domain (2) or (3) and oscillates. As $V_{1}$ is varying in time, we cannot express a simple relationship between the parameters of neuron $N_{2}$ and $V_{1}$, as in equation (4). Nevertheless, oscillations of neurons $N_{1}$ let $V_{1}$ be alternatively positive and negative, which implies that the bifurcation curves of neurons are translated along the abscissa in the plane $(\eta, \epsilon)$ in a periodic manner (the position of saddle points of the cell $N_{2}$ is moved periodically). Thus, the slave neuron $N_{2}$ initially situated in the vicinity of a bifurcation curve may be able to cross sometimes this curve and develop a different dynamical behaviour.

Therefore, the master neuron can control the dy-

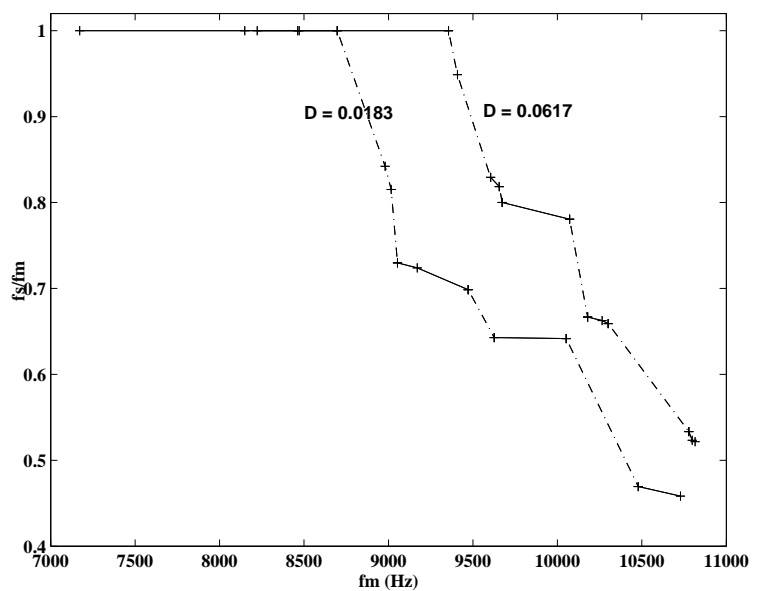

Figure 10: Normalized eigen interspike frequency $f_{s} / f_{m}$ in function of $f_{m}$ for two values of $D$. Parameters : $\varepsilon_{1}=0.1934, \varepsilon_{2}=0.1938$ and $\eta_{2}=0.2163$.

namic of the slave one depending on either its intrinsic behaviour or on the coupling parameter $D$.
The influence of the frequency $f_{m}$ of the oscillating master neuron on the dynamical behaviour of the slave neuron is illustrated in Fig. 10, where the normalised eigenfrequencies $f_{s} / f_{m}$ with $f_{s}$, frequency of the oscillating slave neuron, are plotted in function of $f_{m}$ (given by $\eta_{1}$ as presented in Fig. 6) for two values of $D$.

Initially, the slave uncoupled neuron is so that it lies in domain (2) at the resting state, while the master neuron is oscillating.

In both cases, synchronization occurs for small values of $f_{m}$, the locking range being wider for stronger $D$. When $f_{m}$ is above a critical value depending on the coupling, chaotic behavior emerges, separating frequency locking plateaus. This result suggests that the excitability of the master neuron controls the dynamics of the slave neuron. It is interesting to note that chaotic response is observed in a wide range of frequencies, which in is accordance with numerous biological observations [12].

It also suggests that the coupling parameter $D$ changes the behaviour of the slave neuron.

Note that $D$ is the parameter controlling the inter-

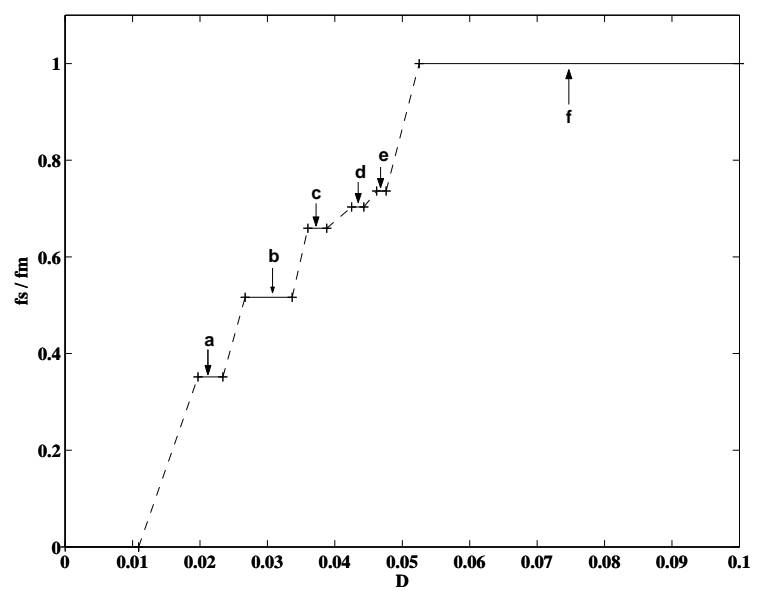

Figure 11: Normalized eigen interspike slave frequency $f_{s}$ by interspike master frequency $f_{m}$ versus $D$ with $\epsilon_{1}=0.1934, \epsilon_{2}=0.1938, \eta_{1}=0.2527$ and $\eta_{2}=0.2262$.

action strength. Then changing $D$ mimics variable "plasticity" of the synaptic interaction. We fix the 
master frequency (hence fix the incoming spike message) and show how the parameter $D$ controls the response dynamics of the slave neuron (Fig. 11 and 12)

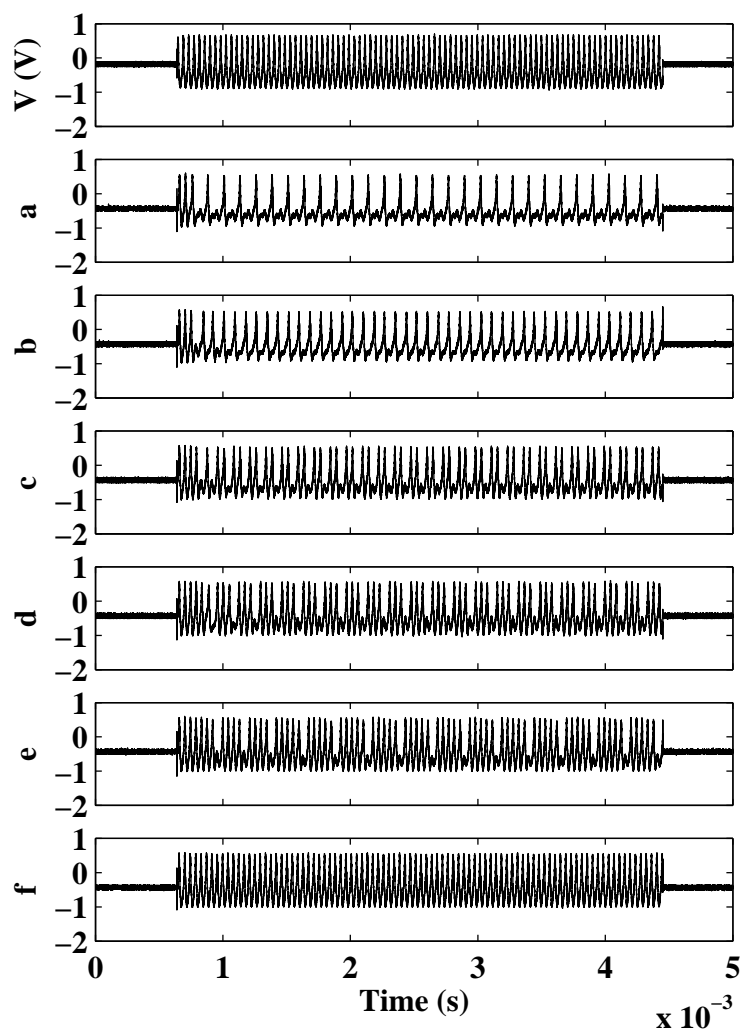

Figure 12: Temporal evolution of experimental voltage $U_{2}$ for different values of $D$ corresponding to cases $(a-f)$ of Fig. 11. Voltage of the master neuron $N_{1}$ is shown on top. Abscissa : $0.5 \mathrm{~ms} / \mathrm{div}$; ordinate: $1 \mathrm{~V} /$ div.

Figure 11 shows normalized spike frequency locking diagram. Spike frequency locking is defined as the ratio $N: M$ where $M$ is the number of master spikes in a certain time window and $N$ is the number of successive response spikes of the slave neuron. Figure 12 (a-f) illustrates the spike trains corresponding to plateaus (a-f) in Fig. 11. With increasing value of $D$ (from a to $\mathrm{f}$ ) the number of response spikes in- creases reaching its maximum value $f_{s} / f_{m}=1$ (Fig. 12 (f) to be compared with the curve at the top corresponding to master oscillations). This corresponds to 1 : 1 spike synchronization mode when the slave repeats the incoming spike message. The frequency
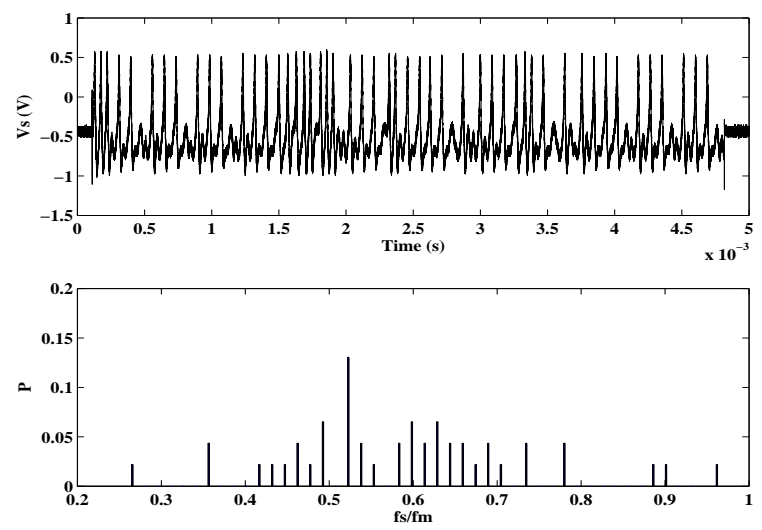

Figure 13: Chaotic signal in the case where $D=$ 0.0535. Abscissa : $5 \mathrm{~s}$ per division; ordinate : $1 \mathrm{~V}$ per division.

locking plateaus are separated by chaotic sequences, represented in dot lines in Fig. 11.

An illustration of a chaotic signal is given in Fig. 13 for $D=0.02775$. The corresponding probability of normalized interspike slave frequency $\mathrm{fs} / \mathrm{fm}$ shows that in a chaotic regime, the interspike frequencies are widely distributed, indicating that the response spike timing can be very complex. In particular, the sequence has variable inter-spike periods which, however, should be associated with the initiating master spike sequence. Then, we define the relative spiking phase variable, $\phi_{n}$, characterizing relative time (phase) shift between a master spike and the following response spike [14]. The definition of $\phi_{n}$ is illustrated in Fig. 14. We take it as

$$
\phi_{n}=\left(t_{n}^{s}-t_{n}^{m}\right) / T,
$$

where $t_{n}^{s}$ and $t_{n}^{m}$ are the spike peak times of the slave and master unit, respectively, $T$ is the master oscillation period and $n$ is the number of successive slave spike in the response sequence. Figure 15 illustrates the spiking phase bifurcation diagram obtained experimentally. Here the value of $\phi_{n}, n=1,2,3, \ldots$ 


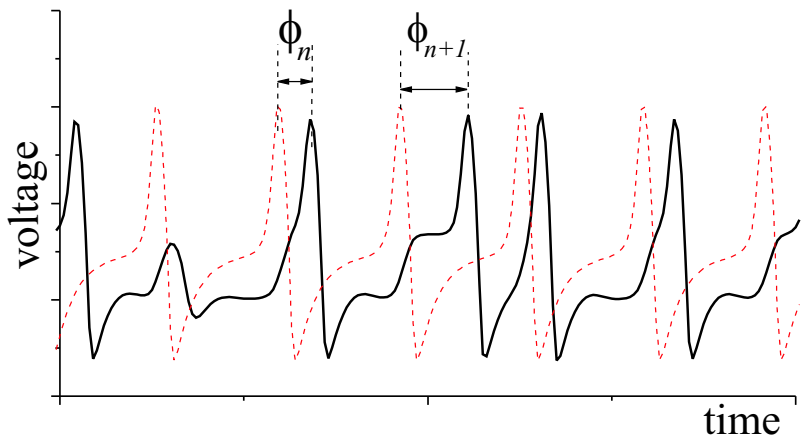

Figure 14: Qualitative view of superimposed master and slave time series shown by dashed and solid curves, respectively. The spiking phase, $\phi_{n}$, is defined as a time shift between master and slave oscillations peaks.

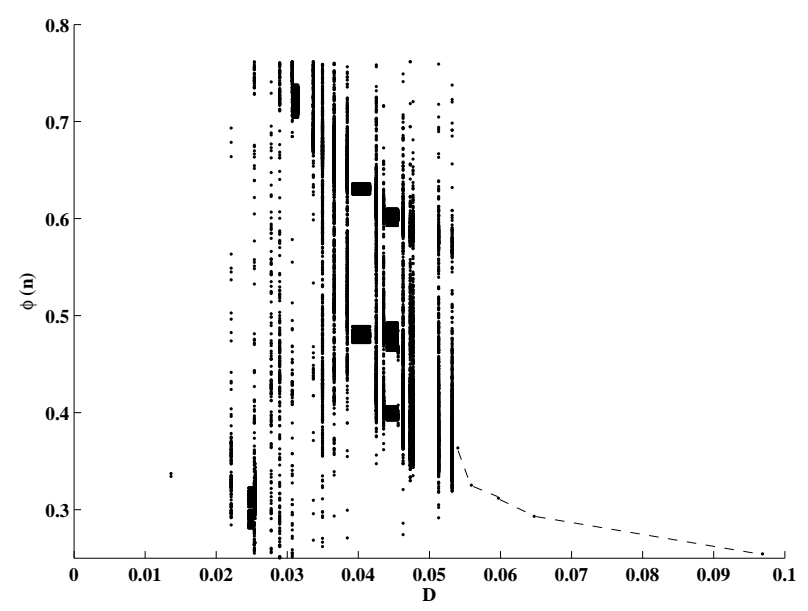

Figure 15: Experimental spiking phase bifurcation diagram. $\phi^{*}$ points are linked by the dot line computed over master-slave experimental traces (Fig. $12,13)$ are plotted versus coupling coefficient $D$. It appears that for 1 : 1 frequency locking mode the phase tends to a single fixed point $\phi^{*}$. This value accounts the phase shift in the synchronized spiking sequence shown in Fig. 13 (f). Note that for more complicated frequency locking modes the phase shift is described either by stable fixed points with $m<\phi^{*}<m+1$, where $m$ is number of "integrated" master spikes to get a response, or by stable periodic orbits of a certain period. Note also that transition to chaos suddenly occurs with decreasing $D$. In this case the spiking phases "fill" some interval with arbitrary changing values from spike to spike. To characterize the phase sequences we calculate the phase map diagrams in the $\left(\phi_{n}, \phi_{n+1}\right)$-plane (Fig. 16). For $1: 1$ locking mode the map has one stable fixed point $\phi^{*}$ (for instance, $\phi^{*}=0.5$ in Fig. 16 (c)). With crossing to the chaotic region the fixed point disappears and the phase starts to oscillate. Note the points are located near some curve in the $\left(\phi_{n}, \phi_{n+1}\right)$-plane. Then the phase dynamics can be approximately described by a $1 \mathrm{D}$ phase map, $\phi_{n} \rightarrow \phi_{n+1}$. Note also that the map curve goes very close to the bisector line, $\phi_{n+1}=\phi_{n}$. Then, the dynamics occurs as follows. The phase shift between master and slave spikes is slowly increasing while evolving near the bisector line. Then, the phase jumps out of the $1: 1$ response region. After one or a few oscillations it returns back to the $\phi_{n+1} \approx \phi_{n}$ - region (Fig. 16 (b)). Such behavior indicates the intermittency chaotic dynamics of the phase variable. Note that the jumps corresponds to the appearance of "missed" spike in the response sequence relative to the master oscillations. More complex intermittency behavior with increasing number of jumps, indicating different missed spike numbers, are shown in Fig. 16 (a).

Thus the response spike sequences obtained in the master-slave neuron model can be associated with spiking phase sequences which can be thought as a kind of neuronal "code". The "code" is tuned by the values of interaction coefficient, $D$. The relative spiking phase represents biologically relevant characteristics of real neuron dynamics. For example, oscillation phase processing plays a crucial role in object tracking by hippocampus neurons [13]. 


\section{Architecture of the neural network}

The previous study allowed us to validate the experimental setup where two neurons where connected unilaterally: The results have been indeed confirmed
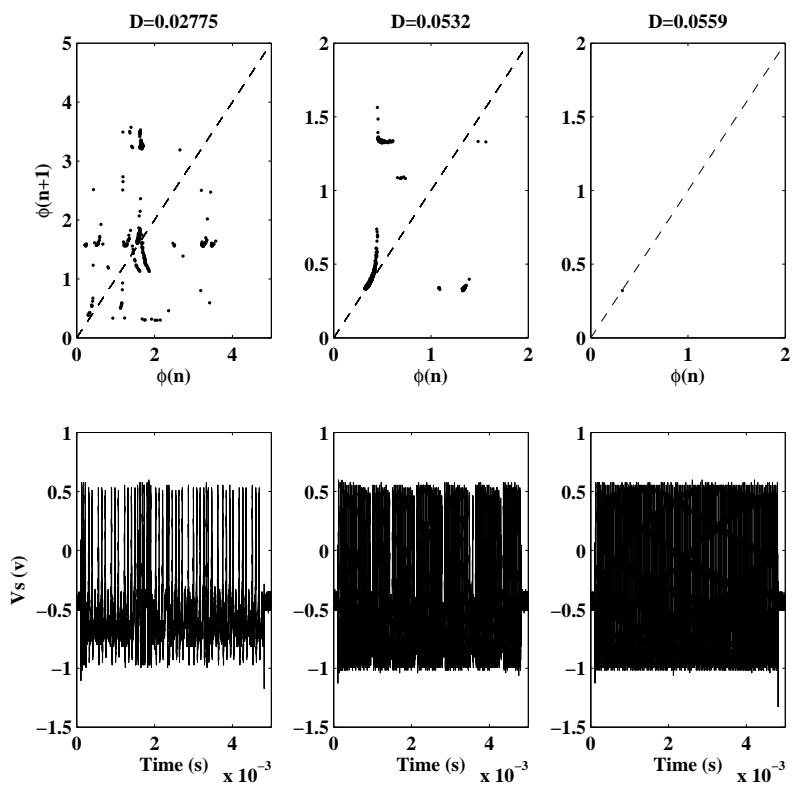

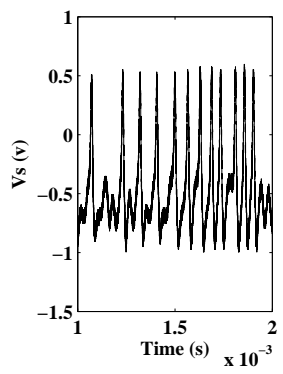

(a)

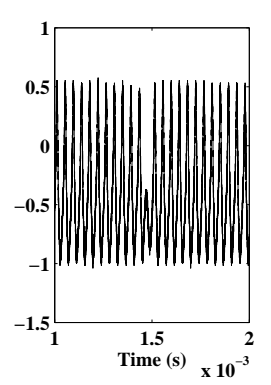

(b)

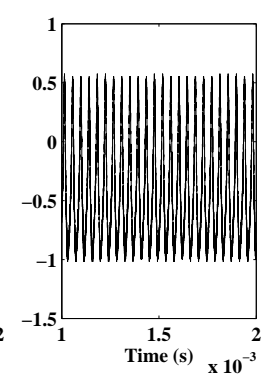

(c)
Figure 16: Experimental $\left(\phi_{n}, \phi_{n+1}\right)$-plane. The phase map are shown at the top of the figure. The corresponding temporal signals are given below. Zoom of each signal is presented at the bottom of the figure. by numeral simulations of eq. (1) using a fourth order runge-kutta algorithm. Therefore, it is interesting to increase the size of the network to realize complex configuration mimicking small assemblies of neurons. In this reductive point of view, the neurons can be connected via unilateral coupling, as presented previously or via bidirectional coupling, which would correspond to electrical synapses (gap junctions). The first mentioned coupling is improved to generate either inhibitory or excitatory connection (see Fig. 17) such as the current flowing from neuron $n$ to neuron $m$ can be expressed by $I=D\left(V_{n}-V c\right)$ where $D$ can be either positive and negative and where $V_{c}$ is the voltage of the neuron $n$ at its resting state. The

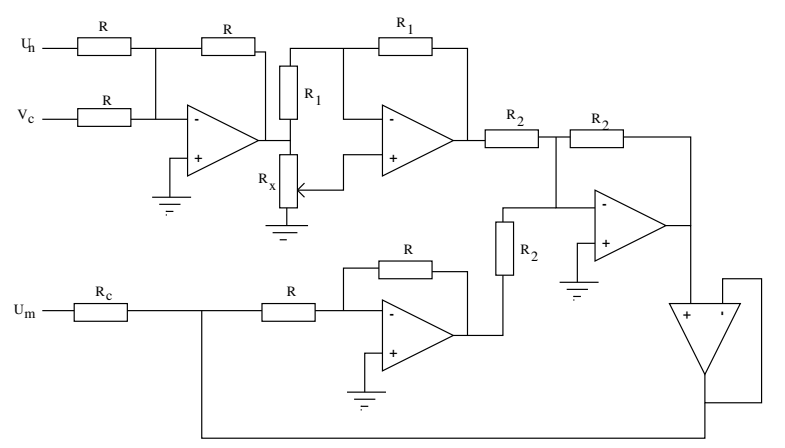

Figure 17: Electrical excitatory or inhibitory coupling.

bidirectional circuit is simply obtained by detuning resistors.

Furthermore, an integration of such a circuit using inductor-like components could be realized, giving this opportunity of very large scale network.

\section{Conclusion}

We have presented analog electronic circuit implementing MFHN mathematical model. It reproduces 
the key features of neuron dynamics including excitability, oscillatory dynamics and bistability. We have experimentally investigated the main bifurcations of the model leading to oscillatory and excitable behavior. We have analysed a simple neuron network architecture with two cells in master-slave configuration, e. g. unidirectional coupling. We have shown that the intervals between successive spikes can be chaotic and depends on the intrinsic behaviour of the master neuron and on the coupling strength. The various dynamics of the slave neuron can be reached by detuning $D$, which could be useful in the point of view of neuronal plasticity.

\section{Acknowledgments}

This research was supported in part by Russian Foundation for Basic Research (grants 03-02-17135), by grant of President of Russian Federation (MK 4586.2004.2). V.B.K. acknowledges Russian Science Support Foundation for financial support.

\section{References}

[1] Hodgkin, L. A. and Huley, A.F., J. Physiol. 117 (1952) 500.

[2] Pinto, R.D., Varona, P., Volkovskii, A.R., Szcs, A., Abarbanel, H.D.I. and Rabinovich, M.I., Phys. Rev. E 62 (2) (2000) 2644.

[3] Lemasson, G., Lemasson S. and Moulins, M., Prog. Biophys. Mol. Biol. 64 (1995) 201.

[4] Fitzhugh, R., Impulse and physiological states in models of nerve membrane, Biophys. J. 50 (1961) $445-466$.

[5] J. Nagumo, S. Arimoto and S. Yoshizawa, An active impulse transmission line simulating nerve axon, Proc. IRE 50 (1962) 2061-2070.

[6] A. C. Scott. Neuroscience: A mathematical primer, Springer-Verlag, New York, 2002.
[7] C. Koch. Biophysics of computation: Information processing in single neurons, Oxford University Press, Oxford, 1998.

[8] S. Binczak, V. B. Kasantsev, V. I. Nekorkin, J. M. Bilbault, Experimental study of bifurcations in modified FitzHugh-Nagumo cell, Electronics Letters 39 (2003) 961-962.

[9] V. B. Kazantsev, Selective communication and information processing by excitable systems, Phys. Rev. E 64 (2001) 056210.

[10] S. P. Dawson, M. V. D'Angelo and J. E. Pearson, Towards a global classification of excitable reaction-diffusion systems, Phys. Lett. A $\mathbf{2 6 5}$ (2000) 346-352.

[11] J. Rinzel and B. B. Ermentrout, Analysis of neural excitability and oscillations, In Methods in neuronal modeling, C. Koch and I. Segev editors, second edition, MIT press, Cambridge, Massachusetts (1998) 251-292.

[12] H. Korn and P. Faure, Is there chaos in the brain ? II. Experimental evidence and related models, C.R. Biologies 326 (2003) 787-840.

[13] J.C. Magee, Trends Neurosci. 26 (1), 14 (2003); O. Melamed et al. Trends Neurosci. 27 (1), 11 (2004).

[14] K. Yoshino, T. Nomura, K. Pakdaman, and S. Sato, Phys. Rev. E 59, 956 (1999); K. Pakdaman, Phys Rev E 63, 041907 (2001). 